\title{
Period doubling and non-linear resonance in the black hole candidate IGR J17091-3624?
}

\author{
P. Rebusco ${ }^{1}$, P. Moskalik² , W. Kluźniak ${ }^{2}$, and M. A. Abramowicz ${ }^{3,2,4, \star}$ \\ ${ }^{1}$ Experimental Study Group, Massachusetts Institute of Technology, 77 Massachusetts Avenue, 02139 Cambridge (MA), USA \\ e-mail: pao@space.mit.edu \\ 2 Copernicus Astronomical Center, ul. Bartycka 18, 00-716 Warszawa, Poland \\ e-mail: [wlodek; pam] @camk.edu.pl \\ 3 Department of Physics, University of Gothenburg, 412-96 Göteborg, Sweden \\ e-mail: marek.abramowicz@physics.gu.se \\ ${ }^{4}$ Institute of Physics, Faculty of Philosophy and Science, Silesian University in Opava, Bezručovo nám. 13, 746-01 Opava, \\ Czech Republic
}

Received 25 January 2012 / Accepted 10 March 2012

\section{ABSTRACT}

\begin{abstract}
The two high frequency quasi periodic oscillations (HFQPOs) recently reported in the black hole candidate IGR J17091-3624 are in a 5:2 frequency ratio $(164 \mathrm{~Hz}$ to $66 \mathrm{~Hz})$. This ratio is strongly suggestive of period doubling and nonlinear resonance analogous to phenomena known in RV Tauri-type pulsating stars (and recently discovered also in oscillations of RR Lyrae-type and of BL Herculistype variables). An interpretation of the frequency ratio in terms of nonlinear interactions and a comparison with the HFQPOs reported in GRS 1915+105 may imply a mass of about six solar masses for the black hole in IGR J17091-3624.
\end{abstract}

Key words. accretion, accretion disks - black hole physics - X-rays: binaries - asteroseismology

\section{Introduction}

The source IGR J17091-3624 has received some notoriety as a possibly low mass $\left(M \sim 3 M_{\odot}\right)$ black hole candidate (Altamirano et al. 2011). The suspected low mass value has been questioned by Altamirano \& Belloni (2012) who discovered two high frequency quasi periodic oscillations (HFQPOs) with frequencies $66 \mathrm{~Hz}$ (at 8.5 sigma) and $164 \mathrm{~Hz}$ (at 4.5 sigma). We point out that the two frequencies are in a 5:2 ratio.

If the underlying fundamental mode of oscillations corresponds to that responsible for the pair of HFQPOs in a $5: 3$ ratio previously observed in another black hole transient (GRS 1915+105), then the black hole in IGR J17091-3624 would have a run-of-the-mill mass, with a value comparable to that of XTE J1550-564 (which is reported to have $9.10 \pm$ $0.61 M_{\odot}$, Orosz et al. 2011). The 5:2 ratio further strengthens the case for non-linear resonance of accretion disk oscillations as the source of HFQPOs.

The resonance interpretation was first suggested by Kluźniak \& Abramowicz (2001a,b), who argued that the QPO phenomenon (van der Klis 2000) cannot be of a kinematic origin but must be due to accretion disk oscillations, and that the twin-peak HFQPOs are due to resonances between particular modes of disk oscillations. These authors (Abramowicz \& Kluźniak 2001) also noticed that the $450 \mathrm{~Hz}$ frequency in the X-ray flux of the black hole candidate GRO J1655-40 discovered by Strohmayer (2000) was in a 3:2 ratio to the previously known $300 \mathrm{~Hz}$ frequency of a HFQPO in the same source.

Subsequently, Kluźniak \& Abramowicz (2002) noted that "a 2:3 ratio [is] in agreement with the 300 and $450 \mathrm{~Hz}$ frequencies reported in GRO J1655-40 and the 184 and $276 \mathrm{~Hz}$

\footnotetext{
* A visitor at Charleston College, SC USA.
}

frequencies reported in XTE J1550-564. [...] a 5:3 ratio [is] in agreement with the 69.2 and $41.5 \mathrm{~Hz}$ frequencies reported in GRS 1915+105", and interpreted the observations in terms of parametric resonance of epicyclic oscillations in general relativity (GR).

Specifically, a rotation supported (slender) fluid torus about a black hole has modes of oscillation with eigenfrequencies corresponding to the radial and vertical epicyclic frequencies in the Kerr metric. In parametric resonance these eigenfrequencies are in a 3:2 ratio, and the first overtone of the vertical mode adds a third frequency for a sequence of ratios of 5:3:2. A more general discussion of possible 3:2 orbital resonances, and the resulting spin estimates for microquasar black holes is given in Török et al. (2005). The epicyclic modes are always among fundamental modes of oscillation of fluid disks and tori around black holes and neutron stars (see, e.g., Blaes et al. 2007; Blaes et al. 2006, and references quoted there). However, as higher frequencies in a 3:2 ratio were subsequently reported in GRS J1915+105 (McClintock \& Remillard 2006; Remillard \& McClintock 2006), the identification of the $69.2 \mathrm{~Hz}$ oscillation with the overtone of parametric epicyclic resonance was abandoned (Kato 2004).

In this Letter, we would like to present a unified scheme for the HFQPOs in IGR J17091-3624 and GRS J1915+105, and their frequency ratios, which is not necessarily tied to any specific theoretical model of accretion disk oscillations.

\section{Period doubling and half-integer frequencies}

We would like to suggest a simple interpretation of the 5:3 and $5: 2$ ratios of the reported frequencies in black hole transients that has a direct analogue in phenomena observed in certain pulsating 
stars. One reason for framing the discussion in this way is that although accretion disks, just like stars, are fluid bodies in (or near) hydrostatic equilibrium, the internal physical properties (such as the distribution of pressure or density) of accretion disks are not well known and this introduces an additional uncertainty in modeling their oscillations.

As already pointed out in the context of HFQPOs (Abramowicz \& Kluźniak 2003), a subharmonic (at half the fundamental frequency) is a hallmark of non-linear interactions. The presence of subharmonics is a frequency domain manifestation of period doubling. In the time domain, it corresponds to oscillations with twice the original period and with alternating cycles of higher and lower maxima and deeper and shallower minima. Such behavior is well known in some oscillating stars. It has been observed for decades in the RV Tauri-type variables. In fact, such alternations of the lightcurve are the very definition of this class of pulsators. A frequency analysis of RV Tauritype stars reveals the presence of subharmonics at $1 / 2$ and at $3 / 2$ of the fundamental oscillation frequency, $f$ (Pollard et al. 1996; Kolláth et al. 1998). Similar subharmonics are also occasionally detected in the W Virginis-type stars (e.g., Templeton \& Henden 2007), which are closely related to the RV Tauri-types variables. In the last two years, period doubling and subharmonics have been discovered in two other classes of pulsators: in the RR Lyrae-type stars (Szabó et al. 2010) and in the BL Herculistype stars (Smolec et al. 2012).

In most cases, more than one subharmonic is present. This is a result of the strong nonlinearity of oscillations, which generates harmonics of the main frequency (at $2 f, 3 f$, etc.), but also odd multiples of the subharmonic (at $3 / 2 f, 5 / 2 f$, etc.). The most extreme example of this behavior is seen in the prototypical variable RR Lyrae, where a complete sequence of subharmonic multiples from $1 / 2 f$ up to $27 / 2 f$ has been detected (Kolenberg et al. 2011). However, BL Her type stars exhibit only a few odd multiples of the subharmonic, in one case the observed sequence being $1 / 2 f, 5 / 2 f, 7 / 2 f, 9 / 2 f$. It is not understood why $3 / 2 f$ fails to show up, but that is often the case in pulsating stars - some, but not all, of the predicted (allowed) frequencies are present.

The period doubling phenomenon in oscillating stars is well understood and is reproduced by state-of-the art numerical hydrodynamical models. For the BL Herculis-type stars it was actually predicted 20 years prior to its discovery (Buchler \& Moskalik 1992). By careful analysis of the models, the origin of period doubling in oscillating stars has been traced to the occurence of a half-integer resonance of the type $f_{k} / f \simeq n+1 / 2$ between the fundamental mode and another oscillation mode of frequency $f_{k}$ (Moskalik \& Buchler 1990).

In the BL Herculis-type stars period doubling and the concomitant subharmonic frequencies are induced by the 3:2 resonance (Buchler \& Moskalik 1992; Smolec et al. 2012), in the RR Lyrae-type stars by the 9:2 resonance (Kolláth et al. 2011), while in the case of the W Virginis and RV Tauri-type variables by the 5:2 resonance (Moskalik \& Buchler 1990) between the fundamental mode and a radial overtone. We note that resonances in such ratios are possible also in accretion disks. Horák (2004) and Rebusco (2004) independently considered geodesic perturbations and indeed found the possibility for a 3:2 ratio and other $m: n$ ratios between vertical and radial epicyclic oscillations. For plane-symmetric resonances $n$ is even, and the $3 \mathrm{rd}$ order resonance occurs when $m / n=3 / 2$, the 5 th order resonance occurs when $m / n=5 / 2$, and so on, with the higher order resonances being less probable.
Taking our cue from pulsating stars, we could assume that the two black hole systems under discussion exhibit various odd multiples of the subharmonic $f / 2$ of their respective fundamental frequencies, $f$. In particular, in GRS 1915+105 one could assume that the two HFQPO frequencies that are in a $5: 3$ ratio correspond to $5 / 2 f$ and $3 / 2 f$, while in IGR J17091-3624 one observes the fundamental $f$, and $5 / 2 f$. Assuming that in both cases the fundamental frequency corresponds to the same mode of oscillations, one can compare the source masses (assuming similar values of the dimensionless spin parameter $a_{*}$ ), as in GR the mode frequencies are inversely proportional to the black hole mass. In this scheme, the fundamental in GRS $1915+105$ would be $2 / 3$ of the lower frequency in the observed pair $69.2 \mathrm{~Hz}$ and $41.5 \mathrm{~Hz}$, i.e., about $28 \mathrm{~Hz}$ - we note that a $27 \mathrm{~Hz}$ frequency was also detected in this source by Belloni et al. (2001). The lower frequency reported in IGR J17091-3624, $66 \mathrm{~Hz}$, is about twice the fundamental frequency inferred for GRS 1915+105, and the same ratio (2.4) is, of course, obtained from the higher reported frequencies, 164 $\mathrm{Hz}$ and $69 \mathrm{~Hz}$. This ratio would imply that the black hole mass of IGR J17091-3624 is about half the mass of the black hole in GRS $1915+105$, which was reported to be $14 \pm 4 M_{\odot}$ (Greiner 2001). Thus, the black hole in IGR J17091-3624 should have a mass of about $6 M_{\odot}$ (modulo the unknown spin).

So far, we have been referring to period doubling in the sense of its purely observational consequences of alternating higher and lower maxima and deeper and shallower minima in the light curve or, equivalently, the presence of subharmonics in the frequency domain. However, the term "period doubling" is used to denote a bifurcation in a dynamical system, a transition from one periodic state to another, period doubled one. In some cases this is followed by a cascade of doublings, which can lead to chaos (and quasi-periodicity). In the case of pulsating stars, numerical models display the period doubling bifurcation. It can also be demonstrated that the original periodic solution looses its stability at the onset of bifurcation. Such an analysis has not yet been performed for accretion disk oscillations, so our suggestion of period doubling in the two black hole systems does not have the strong theoretical underpinning that period doubling has in BL Her, RR Lyr, and RV Tau type stars. One has to be open to the possibility that the 5:2 and 5:3 frequency ratios in IGR J17091-3624 and GRO J1915+105 may be a manifestation of a direct resonance between two modes of oscillation (Kluźniak \& Abramowicz 2001a,b; Abramowicz \& Kluźniak 2002; Török et al. 2005), which does not necessarily lead to period doubling. For instance, a 3:2 coupling will lead to period doubling if the higher frequency mode is resonantly driven by the lower frequency mode (Moskalik \& Buchler 1990), but not vice versa.

\section{Properties of stellar and disk oscillators}

We have referred to three classes of stellar pulsators, RV Tau type stars, RR Lyrae type stars and BL Herculis type stars, in which the growth rates of the oscillations (e.g., thousands of days in RR Lyrae) are much shorter than other characteristic timescales, such as the thermal timescale $\left(10^{8}\right.$ years in RR Lyrae), but much longer than their periods. The amplitudes of the oscillation have reached saturation. Thus the oscillations are often essentially strictly periodic (or multiperiodic). The subharmonic oscillations typically have amplitudes much lower than the harmonics, but they are not always periodic: in RR Lyrae the main oscillation is amplitude modulated and period doubling is present in some stretches of data, but not in others (Szabó et al. 2010; Kolenberg et al. 2011), thus leading to a finite width of the 
subharmonics in the frequency domain. In RV Tauri type stars, the subharmonics also have a finite width.

The excitation and damping mechanism of the accretion disk oscillations (Kato 2001; Wagoner 2002) is without doubt very different from that in the stellar pulsators discussed above. When the QPOs are present the disk is probably in a very turbulent state and varies on all timescales, including scales which are not very much longer than the dynamical timescale. This makes modeling the oscillations a challenging task. If we were to search for similar behavior in stellar systems, we would look to red giants and solar type stars, where short-lived oscillations are stochastically excited by turbulent convection (e.g., De Ridder et al. 2009; Goldreich \& Keely 1977; Houdek 2010). Period doubling has not (yet?) been reported in those stars.

With only two HFQPOs recently discovered in J17091-3624 it is too early to be confident about the presence of a subharmonic series similar to the one observed in the pulsating stars, even though the $5 / 2$ subharmonic is much weaker than the presumed fundamental at $66 \mathrm{~Hz}$. While we have reinterpreted the GRS 1915+105 QPO frequencies in light of the newly discovered ones in J17091-3624, it is important to note that simple harmonics of the presumed fundamental at $27 \mathrm{~Hz}$ have not yet been reported in that source.

The black hole HFQPOs can only be extracted from the data upon integration of the X-ray signal for thousands of oscillation periods, the resulting average Lorentzian profiles fitted to the data have widths comparable to the frequency (quality factor, $Q$, of a few) - it is not clear whether this reflects an intrinsically low coherence of black hole disk oscillations or whether the frequencies shift during the integration time. We note that in neutron stars, where the signal is stronger, at least some HFQPOs have been shown to have a larger coherence, with a quality factor of $Q \approx 200$ (Barret et al. 2005; Mukherjee \& Bhattacharya 2011). These values are not so different from those in oscillating red giants: in $\epsilon$ Oph oscillation modes have periods of $\sim 6 \mathrm{~h}$, with a lifetime of possibly 3 days, for a quality factor value of $Q \approx 10$ (Barban et al. 2007), and in another source, CoRoT 101034881 the corresponding numbers are about $6 \mathrm{~h}$, and 50 days, for a $Q \approx 200$ (De Ridder et al. 2009). In the Sun, the 5 min oscillations have a $Q \sim 10^{3}$, e.g., the $4.9 \mathrm{~min}$ period oscillation has a lifetime of 0.7 days for a $Q=270$, while the $8.2 \mathrm{~min}$ period corresponds to a lifetime of 17.1 days, for a $Q=3000$ (Libbrecht 1988).

\section{Discussion and conclusions}

In this Letter we suggest a possible interpretation of the recently observed HFQPOs in the black hole candidate IGR J170913624. By analogy with stellar oscillations, we interpret HFQPOs in this source (as well as in GRS 1915+105) in terms of nonlinear resonances of oscillating modes of the accretion disk. The present work is not concerned with a specific model for the origin of such oscillations: it simply illustrates universal features of non-linear interactions that match observations, and lead to a reasonable mass estimate (of about $6 M_{\odot}$ ) for IGR J17091-3624 on the assumption that the $66 \mathrm{~Hz}$ oscillation corresponds to the $27 \mathrm{~Hz}$ oscilation in GRS 1915+105. The common denominator between accretion disks and stars is that they are fluid bodies near hydrostatic equilibrium (although with different accuracy: RR Lyrae can be considered stationary on a timescale of $10^{8}$ years, while the state of the disk in a black hole transientcan change in minutes). The fact that in one case GR effects are important while in the other they are not, may be relevant in the discussion of the nature of the fluid oscillations, but it does not affect the simple period doubling phenomenon.

If confirmed, period doubling in black hole QPOs could be the first such detection in a stochastically driven astrophysical system. A more detailed investigation of the full spectrum of QPOs in IGR J17091-3624 is eagerly awaited, as it could reveal a sequence of harmonics and subharmonics with amplitude structure expected in a period doubling system or, alternatively, suggesting the presence of a direct high-order resonance.

Acknowledgements. This work was supported by Polish grants NN203381436 and NCN 2011/01/B/ST9/05439. W.K. thanks the hospitality of the Aspen Center for Physics, where a part of this work was completed.

\section{References}

Abramowicz, M. A., \& Kluźniak, W. 2001, A\&A, 374, L19 Abramowicz, M. A., \& Kluźniak, W. 2003, Gen. Relat. Grav., 35, 69 Altamirano, D., Belloni, T., Linares, M., et al. 2011, ApJ, 742, L17 Altamirano, D., \& Belloni, T. 2012, ApJ, 747, L4

Barban, C., Matthews, J. M., De Ridder, J., et al. 2007, A\&A, 468, 1033

Barret, D., Kluźniak, W., Olive, J. F., Paltani, S., \& Skinner, G. K. 2005, MNRAS, 357, 1288

Belloni, T., Méndez, M., \& Sánchez-Fernández, C. 2001, A\&A, 372, 551

Blaes, O. M., Arras, P., \& Fragile, P. C. 2006, MNRAS, 369, 1235

Blaes, O. M., Šrámková, E., Abramowicz, M. A., Kluźniak, W., \& Torkelsson, U. 2007, ApJ, 665, 642

Buchler, J. R., \& Moskalik, P. 1992, ApJ, 391, 736

Deubner, F.-L., \& Gough, D. 1984, ARA\&A, 22, 593

Goldreich, P., \& Keely, D. A. 1977, ApJ, 212, 243

Greiner, J. 2001 [arXiv: astro-ph/0111540]

Horák, J. 2004, in Processes in the vicinity of black holes and neutron stars, Workshop held at Silesian University, Opava, October 2003 [arXiv: astro-ph/0408092]

Houdek, G. 2010, Astrophys. Space Sci., 328, 237

Kato, S. 2001, PASJ, 53, L37

Kato, S. 2004, PASJ, 56, L25

Kluźniak, W., \& Abramowicz, M. A. 2001a, unpublished [arXiv:astro-ph/0105057]

Kluźniak, W., \& Abramowicz, M. A. 2001b, Acta Physica Polonica B, 32, 3605 Kluźniak, W., \& Abramowicz, M. A. 2002, unpublished [arXiv:astro-ph/0203314]

Kolenberg, K., Bryson, S., Szabó, R., et al. 2011, MNRAS, 411, 878 Kolláth, Z., Buchler, J. R., Serre, T., \& Mattei, J. 1998, A\&A, 329, 147

Kolláth, Z., Molnár, L., \& Szabó, R. 2011, MNRAS, 414, 1111

Libbrecht, K. G. 1988, ApJ, 334, 510

McClintock, J. E., \& Remillard, R. A. 2006, in Compact stellar X-ray sources, ed. W. Lewin \& M. van der Klis, Cambridge Astrophysics Series, No. 39 (Cambridge, UK: Cambridge University Press), 157

Moskalik, P., \& Buchler, J. R. 1990, ApJ, 355, 590

Mukherjee, A., \& Bhattacharyya, S. 2011, ApJ, 730, L32

Orosz, J. A., Steiner, J. F., McClintock, J. E., et al. 2011, ApJ, 730, 75

Pollard, K. R., Cottrell, P. L., Kilmartin, P. M., \& Gilmore, A. C. 1996, MNRAS, 279, 949

Rebusco, P. 2004, PASJ, 56, 553

De Ridder, J., Barbau, C., Baudin, F., et al. 2009, Nature, 459, 398

Remillard, R. A., \& McClintock, J. E. 2006, ARA\&A, 44, 49

Smolec, R., Soszyński, I., Moskalik, P., et al. 2012, MNRAS, 419, 2407

Strohmayer, T. E. 2000, ApJ, 554, L169

Szabó, R., Kolláth, Z., Molnár, L., et al. 2010, MNRAS, 409, 1244

Templeton, M. R., \& Henden, A. A. 2007, AJ, 134, 1999

Terlevich, R. 1992, in Relationships between Active Galactic Nuclei and Starburst Galaxies, ed. A. V. Filippenko, ASP Conf. Ser., 31, 13

Török, G., Abramowicz, M. A., Kluźniak, W., \& Stuchlík, Z. 2005, A\&A, 436, 1

van der Klis, M. 2000, Encyclopedia of Astronomy and Astrophysics, ed. P. Murdin, 2380

Wagoner, R. V. 2002, ApJ, 578, 63 